\title{
Viewpoint
}

\section{Holey Intrinsic Photoconductivity}

\author{
Guido Pupillo and Fabio Mezzacapo \\ IPCMS (UMR 7504) and ISIS (UMR 7006), Université de Strasbourg and CNRS, Strasbourg, \\ France
}

Published February 19, 2013

\begin{abstract}
A new experiment reveals the different dynamics of particles and holes in ultracold fermionic gases in an optical lattice, opening the way to investigate condensed matter transport phenomena such as photoconductivity via quantum optical systems.
\end{abstract}

Subject Areas: Atomic and Molecular Physics, Optics

\author{
A Viewpoint on: \\ Intrinsic Photoconductivity of Ultracold Fermions in Optical Lattices \\ J. Heinze, J. S. Krauser, N. Fläschner, B. Hundt, S. Götze, A. P. Itin, L. Mathey, K. Sengstock, and C. Becker \\ Physical Review Letters 110, 0853022013 - Published February 19, 2013
}

When a crystal absorbs photons of suitable energy, electron-hole excitations are created in pairs leading to an increase in conductivity that is proportional to the photon flux. Such a phenomenon in which light generates electrical current, turning a nonconductor into a conducting material, is known as photoconductivity. While photoconductivity occurs, in principle, in any material, a substantial photocurrent is particularly easy to generate in semiconductors because of their small band gaps. Photoconductivity is of crucial interest for investigating electron-hole dynamics, transport properties of complex compounds, possibly displaying novel physics, as well as for its technological applications like semiconductor photodiodes and photoresistors. However, solid-state systems can be complicated because of the density of constituent atoms, so there is a desire to study physical effects from condensed matter physics in well-controlled analogous systems, such as cold atomic gases, as well as to understand how particle-hole dynamics operate in the latter case.

Writing in Physical Review Letters, Jannes Heinze of the University of Hamburg, Germany, and collaborators present results from combined experimental and theoretical work on the excitation dynamics of an ultracold fermionic gas trapped in a periodic optical potential or "optical lattice" [1]. The experiment is specifically designed to mimic the phenomenon of photoconductivity in an atomic gas. In this experiment, particles are transferred by modulations of the lattice amplitude from the lowest band of the lattice to the second excited one, leaving holes behind. An external harmonic potential induces oscillations of both particles and holes, corresponding to transport in the condensed matter system, and the ensuing dynamics is monitored via momentum-resolved absorption imaging techniques. The parallel with semiconductors is transparent and very appealing: photons

DOI: $10.1103 /$ Physics.6.19

URL: http://link.aps.org/doi/10.1103/Physics.6.19
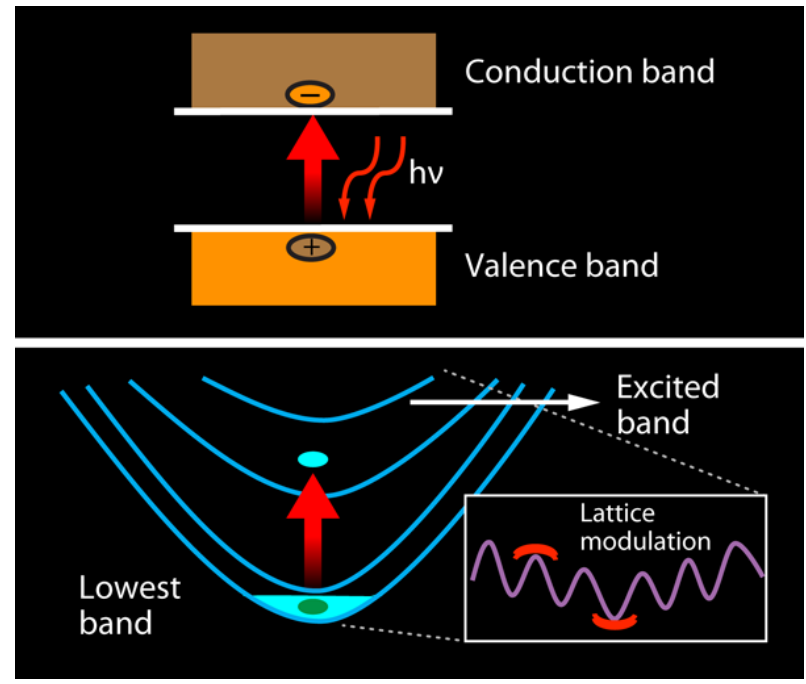

FIG. 1: Excitation process leading to photoconductivity in a condensed matter system (upper panel) and its cold-atom counterpart (lower panel). In the condensed matter system, incoming laser light $(h v)$ excites an electron to the valence band, leaving a positively charged hole behind. In the coldatom analogy, the optical lattice is tuned to allow an atom to move into the excited band, also leaving a hole behind. (APS/Alan Stonebraker)

in semiconductors are equivalent to lattice modulations, electrons are played by fermionic atoms, while holes remain... holes. Figure 1 illustrates the excitation process as occurring in the condensed matter system (upper panel) and in its cold-atom counterpart (lower panel). In the spirit of quantum simulations, one goal is to exploit these analogies to investigate the physics of semiconductors using quantum optical systems.

The research team uses, for the most part, an ultracold 
gas of spin-polarized noninteracting potassium-40 $\left({ }^{40} \mathrm{~K}\right)$ fermionic atoms. In a few cases, a mixture of two different spin states is utilized instead, adding the possibility to include and tune interactions using a Fano-Feshbach resonance (i.e., a resonance with a molecular state that allows for the control of the sign and strength of interspecies two-body interactions). Modulation of the amplitude of the optical lattice promotes, without transferring quasimomentum (i.e., an intrinsic quantum number arising from the translational symmetry of the lattice), a few particles from the lowest to the upper band. However, in contrast to semiconductors, the two bands are curved here because of the presence of a confining harmonic potential - the optical dipole trap. The resonance frequency for excitations thus becomes quasimomentum-dependent because of the different curvatures of the bands, which allows for full control of the initial quasimomentum of the excited particles. Their dynamics can be then monitored using adiabatic band mapping and absorption imaging after a characteristic time-of-flight of 15 milliseconds (ms). Rather than just doing transport measurements, this method allows the team to follow the periodic dynamics of the atoms and resolve their momentum completely. A surprising new feature of this experiment is that here, essentially the same is done for holes: differential absorption imaging techniques are crucial, in this case, to follow the time evolution of the hole depth (i.e., the hole momentum with respect to the Fermi one).

The dynamics of the fermions in the excited band displays extended oscillations in momentum space. The oscillation frequencies increase with that of the harmonic confinement, decreasing instead with the quasimomentum of the excitation as well as with the lattice depth. These oscillations are quite long-lived, with typical lifetimes of the order of $100 \mathrm{~ms}$. Conversely, the hole depth appears almost completely reduced after a much shorter time (i.e., approximately $2 \mathrm{~ms}$ ). However, and this is one of the central results, the hole depth displays a series of periodic revivals whose lifetime is longer as the depth of the optical lattice decreases. The direct momentumresolved measurements of the hole dynamics, and especially of revivals of the hole depth, are an exciting new result whose observation is made possible by the exceptionally well-controlled experimental setup used by the Hamburg team.

For spin-polarized particles, the dynamics has a Hamiltonian description that is essentially determined by the combined effects of the confining periodic and harmonic trapping potentials. The former determines the band structure, with characteristic width $4 J$ for the lowest band (with $J$ being the single-particle tunneling matrix element), while the latter fixes its curvature ( $\nu$ in text), that is, the energy cost paid when a particle moves from the center of the trap to its nearest neighboring lattice site. For the large ratios $(4 J / \nu) \gg 1$, realized in the Hamburg experiment, two classes of eigenmodes are present and can be classified according to their energy $(\epsilon)$. Low-energy modes (with $\epsilon<4 J$ ) are well described by harmonic oscillator eigenstates and, as a consequence, are delocalized around the center of the trap. When populated by fermions, these modes allow for transport and undamped oscillations, as observed in the experiment. Deviations from the harmonic-oscillator spectrum occur for $\epsilon \sim 4 J$ and are due to corrections arising from the lattice potential. These corrections are responsible for dephasing of dipole oscillations in the trap, as previously observed with bosons only [2] High-energy modes $(\epsilon>4 J)$, carefully avoided in the experiment, are instead close to position eigenstates, i.e., localized on either side of the harmonic potential.

Such a peculiar single-particle spectrum, which has an analytic solution in the tight-binding limit [3, 4, can be used to gain a qualitative understanding of the dynamics of both particles and holes. In particular, the surprising revival of the hole depth may be explained with cycles of dephasing and rephasing of the fermionic cloud in the lowest band. The dephasing is much less pronounced for particles in the upper band because of their smaller number as well as the larger bandwidth. For a quantitative comparison to experimental results, the Hamburg team has chosen a particularly elegant theoretical approach, based on a semiclassical treatment [5-7]: when interband transitions can be neglected, the dynamics in each band is shown to map into that of a nonlinear pendulum, whose equation has swapped position and momentum. This treatment fully explains the observed dynamics of both particles and holes, here regarded as particles with negative mass, as standard in condensed matter. Specifically, the fast decay and revival dynamics is due to a larger spread of momentum in phase space for the holes, as a result of the smaller width of the lowest band.

So, having understood this beautiful physics, what now lies ahead? In their work, the authors give us a hint by presenting results for the lifetime of particles in the second band as a function of the scattering length in a mixture of atoms in two different spin states. The lifetime is shown to be strongly dependent on the interspecies interactions and is qualitatively explained in terms of interaction-induced particle-hole recombination. In contrast to traditional condensed matter systems, this interaction is here fully tunable. Combined with the long lifetime of the system demonstrated in this experiment, this capability of tuning interactions may open the way to the investigation of novel fundamental phenomena, allowing for a precise determination of the role of interactions in hole dynamics and lifetime, both in atomic and condensed-matter-type systems, relevant, e.g., to photoconductivity. Since their theoretical treatment would be hardly achievable using current techniques, this class of experiments would constitute a novel example of a "useful quantum simulation" [8]. Applications of quantum simulators are now thrilling the whole physics community, and thus, as in the best stories, it seems that the very best is yet to come. 


\section{References}

[1] J. Heinze, J. Krauser, N. Fläschner, B. Hundt, S. Götze, A. P. Itin, L. Mathey, K. Sengstock, and C. Becker, "Intrinsic Photoconductivity of Ultracold Fermions in Optical Lattices," Phys. Rev. Lett. 110, 085302 (2013).

[2] C. D. Fertig et al., "Strongly Inhibited Transport of a Degenerate 1D Bose Gas in a Lattice," Phys. Rev. Lett. 94, 120403 (2005).

[3] M. Aunola, "The Discretized Harmonic Oscillator: Mathieu Functions and a New Class of Generalized Hermite Polynomials," J. Math. Phys. 44, 1913 (2003).

[4] A. M. Rey, G. Pupillo, C. W. Clark, and C. J. Williams, "Ul- tracold Atoms Confined in an Optical Lattice Plus Parabolic Potential: A Closed Form Approach," Phys. Rev. A 72, 033616 (2005).

[5] L. Pezzè et al., "Insulating Behavior of a Trapped Ideal Fermi Gas," Phys. Rev. Lett. 93, 120401 (2004).

[6] A. R. Kolovsky, and H. J. Korsch, "Bloch Oscillations of Cold Atoms in Optical Lattices," Int. J. Mod. Phys. B 18, 1235 (2004).

[7] C. Hooley and J. Quintanilla, "Single-Atom Density of States of an Optical Lattice," Phys. Rev. Lett. 93, 080404 (2004).

[8] J. I. Cirac and P. Zoller, "Goals and Opportunities in Quantum Simulation," Nature Phys. 8, 264 (2012).

\section{About the Authors}

\section{Guido Pupillo}

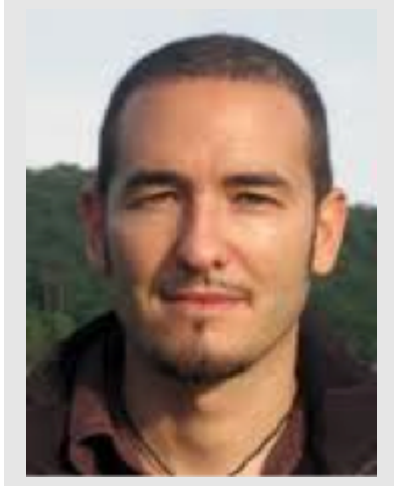

Guido Pupillo is Professor of Physics at the Université de Strasbourg and Director of the Quantum Physics Lab at the Institutes IPCMS and ISIS in Strasbourg, France. After receiving his Ph.D. from the University of Maryland in 2005, for research conducted at NIST, Gaithersburg, he worked at the Austrian Academy of Sciences and at the University of Innsbruck, Austria, where he received his Habilitation in 2011. He is the recipient of the prestigious ERC St-grant 2012 and the French ANR Chair d' Excellence 2012. His group carries out research on atomic, molecular, and optical physics, and nonequilibrium dynamics of quantum systems.

\section{Fabio Mezzacapo}

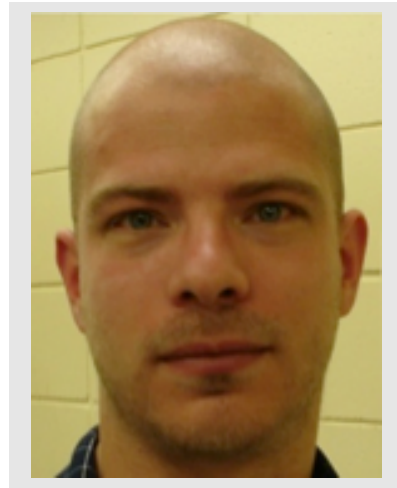

Fabio Mezzacapo is a Senior Postdoctoral Researcher at the Université de Strasbourg, France. He received his Ph.D. in physics from the University of Alberta, Canada, in 2008. Until 2012, he worked at the Max Planck Institute for Quantum Optics in Garching, Germany. His main current research interests are centered on strongly correlated 2D fermionic systems and quantum gases. 